\title{
Redes globales de innovación ${ }^{1}$
}

\section{Global Networks of Innovation}

\author{
Carmen BUENO CASTELLANOS \\ Universidad Iberoamericana (México) \\ carmenbuenocastellanos@yahoo.com.mx
}

Recibido: 18 de diciembre de 2011

Aceptado: 28 de mayo de 2012

\begin{abstract}
Resumen
El propósito de este artículo es analizar procesos globales en lo local. Por ello se presenta la etnografía de uno de los íconos de la globalización: Las cadenas de producción desterritorializadas. En particular los múltiples y complejos procesos a través de los cuales las firmas globales generan innovaciones. Se analiza el margen de maniobra de las filiales localizadas en México para propiciar actividades de colaboración a nivel local para producir nuevo conocimiento y ser disparadoras de un ambiente innovador. Así mismo se analizan, los recursos y las vinculaciones de las pequeñas empresas nacionales para insertarse a estas cadenas globales y aportar productos que incorporen innovaciones.
\end{abstract}

Palabras clave: innovación, firmas globales, empresas locales, desarrollo regional.

\begin{abstract}
This essay aims to analyze global processes in the local. For this purpose, it presents the ethnography of one of the icons of globalization: De-territorialized production networks. In particular, complex and multiple processes through which global firms generate innovations. It analyzes the leeway the subsidiaries of this firms localized in Mexico have, to bring out collaborations at the local level geared toward the production of new knowledge and trigger innovation environments. Also it points out, national small enterprise's resources and linkages to participate in these global networks and contribute with products that incorporate innovations.
\end{abstract}

Keywords: innovation, global firms, local enterprises, regional development.

SUMARIO: 1. Introducción. 2. Herramientas conceptuales. 3. Metodología. 4. Breve ubicación contextual. 5. El caso de un armadora automotriz. 6. El caso de una abastecedora mundial de componentes automotrices. 7. Una empresa mexicana abastecedora de moldes para firmas globales. 8. A manera de reflexión. 9. Referencias bibliográficas.

${ }^{1}$ Este artículo recoge avances de investigación del proyecto Redes Dinámicas de Innovación integrado por investigadores y estudiantes de la UIA, UAM-C y UNAM con el apoyo de CONACyT. 
Referencia normalizada: Bueno Castellanos, C. (2012) Redes globales de innovación. Revista de Antropología Social, 21: 93-116.

\section{Introducción}

Los estudios sobre la globalización abarcan procesos muy variados. Su comprensión integral no puede basarse en conceptos unificados. Los teóricos de este tema, desde la década de 1990, dan cuenta de manera reiterativa de su complejidad; de las combinaciones, fusiones, mezclas de procesos aparentemente opuestos e incompatibles, como homogeneización y fragmentación; globalización y localización; universalismo y particularismo, que son el resultado de las múltiples conexiones y los infinitos flujos de recursos tangibles e intangibles (Appadurai, 1996; Featherstone, 1997; Hannerz, 1996; Long, 1996; Robertson, 2003). Los términos y enfoques de lo global evocan múltiples causas, agendas y capas históricas (Tsing, 2005: 458). Una de las agendas de los estudios de la globalización, tiene que ver con el reconocimiento de la superioridad y universalidad del proyecto de modernidad ${ }^{2}$ en busca del orden progresivo, el control y la unificación de la naturaleza y la vida social, respaldado por la lógica capitalista, desdoblando un fuerte aparato científico y un determinismo tecnológico que ha acompañado las transformaciones económicas en el plano global (Featherstone, 1997: 72-73). No obstante "La concepción de modernidad como una civilización expansiva, conlleva asimetrías globales que si bien se han difundido por todos lados, o se han sentido en todas partes, es un hecho de que su presencia se reproduce en condiciones variables"(Hannerz, 1996:55)

En este artículo se presenta la etnografía de uno de los íconos de la globalización/ modernidad: las cadenas de producción desterritorializadas que conforman sistemas tecnológicos meticulosamente sincronizados [tightly coupled systems] (Batteau, 2010). En particular, dar cuenta de la fuerza homogeneizadora de los sistemas de producción globalizada y de su imposición y dominación planetaria, que simultáneamente genera vinculaciones selectivas con el aparato productivo local. Esto último da cuenta de la generación de interdependencias asimétricas, intensificando el flujo de recursos del centro a la periferia y no a la inversa. Anna Tsing (2005: 455456) propone analizar los compromisos que estas interconexiones globales tienen con la sociedad. La globalización para la autora es un conjunto de proyectos con especificidades y limitaciones culturales e institucionales que engloban prácticas sociales, infraestructura material, negociaciones y relaciones de poder que, cuando se estudian desde el prisma de lo local dan cuenta de lo que los proyectos de la globalización hacen por el mundo.

Estas cadenas productivas no sólo determinan la conformación de la división internacional del trabajo sino además, controlan la generación y la difusión del

${ }^{2}$ Otro proyecto que se ha generado de manera simultánea es lo que se le ha denominado posmodernidad. Featherstone haciendo referencia a Maffesoli (1997: 120) define a la posmodernidad por el tránsito de la racionalidad a la emocionalidad. El cultivar sentimientos profundos y experiencias sensoriales. Esto ha dado cabida al surgimiento de construcciones sociales efímeras, llamada por los autores como neo-tribus. 
recurso más estratégico en los tiempos actuales: la innovación. Esta última incluye una gama de nuevo conocimiento, que van desde transformaciones estratégicas (innovaciones disruptivas) hasta modificaciones (innovaciones incrementales) tanto en proceso como en producto. La producción global en el siglo XXI se distingue por dar prioridad a la innovación como elemento diferenciador y fundamental para la permanencia exitosa en un mercado altamente competitivo a escala planetaria. La propuesta conceptual de la cual se parte es que innovar es un proceso en el cual intervienen diferentes actores sociales así como diversas instituciones (firmas, empresas locales, clientes, proveedores, institutos de investigación y universidades ubicadas en diferentes partes del mundo), que requieren colaborar para compartir proyectos, información, tecnología, inversiones, aprendizajes e incluso saberes adquiridos en la práctica. A lo largo de las diversas actividades orientadas a generar innovaciones se pueden percibir tensiones y sinergias que obstaculizan o bien posibilitan el fluir de los diversos recursos tangibles e intangibles que se necesitan para la generación de conocimiento ${ }^{3}$.

¿Qué tan estratégico es para las firmas globales instaladas en México participar en proyectos dirigidos a generar innovaciones? Para contestar esta pregunta hay que comprender la lógica operativa de la firma global que tiene una estructura interna que se desdobla en unidades localizadas a todo lo largo y ancho del planeta y que a su vez, teje una red de proveedores especializados con los que comparten marcos regulatorios, normas, rutinas y relaciones de confianza construidas con el tiempo, desarrollando y concentrando un capital social a escala internacional, gestando planes a largo plazo, accediendo a conocimiento codificado y conocimiento tácito estratégico, compartiendo recursos y tecnología. Es un sistema autocontenido con un conjunto de formas institucionales multi-escala (Asheim, 2006), que tiene como finalidad optimizar su productividad e implementar mejoras continuas e innovaciones estratégicas en producto, procesos, tecnologías y materiales que permitan reaccionar a las presiones de un mercado igualmente globalizado.

Esta estructura ha generado su propia dinámica. Su gobernabilidad está dirigida y moldeada de manera supranacional. Para poder sincronizar todos los movimientos, han desarrollado complejas plataformas virtuales que facilitan el flujo de recursos de diversa índole. En la práctica, sólo un grupo selecto inner circle puede acceder a la información privilegiada de esta estructura en red. Se mantiene gran hermetismo, tejiendo circuitos cerrados, en donde las decisiones están centradas en nodos estratégicos a la vez que negociadas de manera multidireccional.

La inercia de esta estructura afecta los vínculos de cooperación a nivel local pues dependen de la estrategia global, que particulariza y relativiza las relaciones en el entorno inmediato. En el caso de México, como se verá más adelante, ha generado ambientes de cooperación débiles o marginales a los programas prioritarios de este sistema global porque generalmente las plantas de las firmas globales en México

${ }^{3}$ Esta conceptualización del término innovación es el que orienta un proyecto interdisciplinario titulado "redes dinámicas de innovación" en el que participamos diversos investigadores de la UNAM, UAM-Cuajimalpa y Universidad Iberoamericana. 
dependen principalmente de la obtención de licencias tecnológicas del extranjero o de importar equipo y tecnología "off-the-shelf". Esto limita e incluso inhibe las posibilidades de derrama de conocimiento estratégico de estas firmas globales a nivel regional/local.

Las preguntas que se contestan en este artículo son: ¿Cuál es la manera en las que las filiales de firmas globales instaladas en México intervienen en procesos orientados a la innovación? ¿Qué posibilidades tienen las empresas pequeñas mexicanas de vincularse en prácticas orientadas a generar innovación con firmas globales? Desde la perspectiva de los actores, analizar si la vinculación firma global-empresa local ha sido un disparador de un ambiente innovador en la región donde están instalados. Para ello en primer lugar se presentarán los conceptos que permiten estudiar las formas de generación de innovaciones en la producción globalizada, posteriormente se aporta la metodología utilizada y una breve ubicación contextual del fenómeno a estudiar. A continuación se desarrolla la información de dos filiales de firmas globales y una pequeña empresa mexicana productora de moldes y troqueles ${ }^{4}$, en particular su lógica de vinculación para generar procesos de innovación.

\section{Herramientas conceptuales}

Hay una serie de conceptos que permite responder a las preguntas propuestas en la introducción. Estos conceptos tienen que ver con los procesos que vinculan en un espacio local, prácticas que responden a la lógica de producción global y en específico a las articulaciones globales/locales generadoras de actividades orientadas a la innovación.

Como se mencionó anteriormente, innovación hoy día es un término que está en el centro de la agenda de la sociedad del conocimiento. Esta discusión no es nueva. En la primera mitad del siglo XX, Schumpeter (1957) en un ambiente económico que buscaba "la prosperidad", hizo hincapié en la importancia de que el empresariado asumiera los riesgos y percibiera los beneficios de estimular innovaciones tanto técnicas como organizativas para hacer frente a un medio altamente competitivo. Para este autor, innovar consistía en la investigación científico-tecnológica que culminara con la puesta de un producto en el mercado. Su propuesta está básicamente centrada en el perfil del empresario y como una actividad llevada a cabo de manera endógena al interior de la empresa, cuya finalidad era agregar valor a la

${ }^{4}$ Los moldes y los troqueles tienen una importancia estratégica para la producción industrial. Ambos dos coinciden en ser herramientas de altísima precisión, necesarias para elaborar cada una de las piezas o partes de un artefacto, desde lo más simple como una cubeta hasta lo más complejo como un avión o un automóvil. Estas herramientas han evolucionado significativamente y revisten una gran complejidad y variedad tecnológica. Un productor de estos herramentales tiene que tener un amplio conocimiento de las propiedades de los metales que se utilizan para fabricarlos, de su resistencia, elasticidad, plasticidad, deformación, estructuras moleculares, composiciones, así como de la complejidad de las piezas y de la materia prima que se va a intervenir. La diferencia grosso modo es que el molde sirve para formar piezas cuya materia prima es líquida como sería el plástico y el hule y se vacía o inyecta en herramentales que tienen múltiples cavidades, mientras que el troquel modifica la estructura y el volumen de metales mediante la aplicación de una carga. 
producción y no hacia la búsqueda de un bien común. Esta definición en la actualidad ha tomado un matiz de mayor complejidad en la cual, la innovación se explica como un proceso evolutivo y dinámico que tiene lugar en un espacio multi-escala ${ }^{5}$, que se nutre y es resultado de interacciones con diversos agentes sociales. Es por ello que la definición que prevalece en este artículo se centra en la capacidad de las empresas de generar vínculos sistémicos e interactivos con firmas y empresas pero también con diversas instituciones que aporten información, insumos materiales, experiencia, saberes que apoyen al desarrollo tecnológico y organizativo de las actividades productivas ${ }^{6}$. En este sentido, las empresas son vistas como organizaciones que aprenden, mezclando bases específicas de conocimiento tácito y codificado. Esta mezcla de conocimiento se aplica en pruebas de experimentación, simulación o trabajo práctico, en un proceso de "learning by doing" o "learning by interacting" y que está dirigido a la eficiencia y fiabilidad de la relación cliente-proveedor de productos que agregan valor por la importante complejidad tecnológica que contienen.

La generación de espacios socialmente construidos para la innovación están inmersos en múltiples procesos en los que lo global teje puntos de intersección e incluso se traslapa con lo local, gestando una nueva geografía económica al producir nuevas espacialidades y temporalidades (Sassen, 2001). Siguiendo la propuesta analítica de Saskia Sassen, la globalización económica es estratégica y por tanto selectiva; siendo las firmas globales las que dictan la institucionalidad, normas y regulaciones de los circuitos económicos globales. Estos últimos se distinguen por la dispersión y desconcentración de los procesos de producción en diversos territorios a nivel mundial, al mismo tiempo que centralizan las funciones regulatorias en puntos estratégicos cuyo rol es el control de la producción desterritorializada. Es en este sentido, que se produce una nueva espacialidad. El desarrollo tecnológico está al servicio de la lógica de este nuevo orden de la geografía económica mundial, sometiendo a la producción a ritmos cada vez más acelerados de respuesta, gestando nuevas temporalidades. Para explicar el impacto que esto tiene en lo local, la autora propone el concepto de zonas de frontera (Ibid., 273) que son los puntos de intersección de la producción local con la dinámica global. Son espacios de tensión entre formas organizativas que operan con regulaciones y recursos disponibles en lo local, al mismo tiempo que, se les exige reaccionar a las espacialidades y temporalidades globales. En este sentido, las filiales de firmas globales como las empresas locales están "incrustadas" en esta red global de espacios locales en la que se ven obligadas a responder a la velocidad y a la institucionalidad de la producción global.

${ }^{5}$ Esta propuesta ha inspirado múltiples modelos de análisis de la innovación, algunos que atienden la generación de sistemas regionales o nacionales de innovación. (Algunas referencias clásicas sobre esto son Charles Edquist, 2004; Lundvall, 1992) y otros que se centran en la vinculación entre las empresas, las universidades y el Estado, integrando una “triple hélice". (Leydesdorff, H., Etzkowitz, H., 1996).

${ }^{6}$ Esta propuesta es ampliamente compartida por investigadores mexicanos. Algunas referencias a consultar son los libros coordinados por Casas y Luna (2000), Villavicencio y López de Alba (2009), Dutrénit (2009). 
Una de las problemáticas centrales que permiten analizar empíricamente las interacciones que concurren en las zonas de frontera a las que se refiere Sassen, es la derrama de conocimiento. En México se han desarrollado estudios que analizan, estas derramas locales/regionales como uno de los impactos significativos de la presencia de las firmas globales ${ }^{7}$. Al respecto, el argumento expuesto por Dutrénit y Fuentes (2009) es que las firmas globales portan consigo capacidades tecnológicas y organizacionales de la casa matriz. Esto le da a la filial instalada en el país, una ventaja competitiva y capacidad de generación de conocimiento que después es transferida a las empresas locales. Para analizar este fenómeno, las autoras aluden a varios mecanismos de derrama de conocimiento: demostración-imitación, movilidad de capital humano, competencia derivada de la estructura de mercado, vínculos extranjeros obteniendo una mayor propensión para exportar, vínculos de proveeduría, entrenamiento, y transferencia tecnológica directa ${ }^{8}$.

Ahora bien, esta derrama de conocimiento tiene que contar con empresas locales que tengan no sólo la infraestructura adecuada sino además, la capacidad organizativa necesaria para estimular procesos de absorción de conocimiento. Para poder hacer un estudio de corte cualitativo resulta central indagar sobre las diferentes modalidades en la que la derrama de conocimiento opera como para incentivar procesos de aprendizaje a través de la vinculación ${ }^{9}$. Para analizar esto, habría que probar el alcance del concepto de capacidades dinámicas de Teece y Pisano (1994). Estos autores argumentan que la estrategia empresarial basada en recursos, sobre todo en la acumulación de activos tecnológicos no ha demostrado ser una alternativa exitosa en el mercado, sino más bien el uso de competencias funcionales de una organización para aprender, adaptarse, cambiar y renovarse ante las presiones del entorno. Habría que ponderar esta discusión pues los autores seguramente elaboraron este concepto, al estudiar firmas que se distinguen por ver a la tecnología como una panacea, invirtiendo permanentemente en infraestructura que muchas veces está subutilizada. En este ensayo, será útil el concepto de capacidades dinámicas en tanto permita analizar la creatividad de los actores sociales para reaccionar a la escasez de recursos, como es el caso de las empresas locales.

Los conceptos antes expuestos acerca de la vinculación firma global-empresa local presupone no sólo la importancia de la derrama económica que esto implica, sino la necesidad de generar un ambiente empresarial a nivel local capaz de reac-

${ }^{7}$ Gabriela Dutrénit y Claudia de Fuentes (2009) coordinaron un estudio comparativo de pymes de moldes y troqueles, en donde aplicaron una encuesta para estudiar concretamente la capacidad de absorción de estas pymes de la derrama de conocimiento de firmas globales.

${ }^{8}$ Una exhaustiva revisión de este tema se presenta en el capítulo del libro escrito por Gabriela Dutrénit y Claudia de Fuentes "Derramas de conocimiento y capacidades de absorción" en Sistemas regionales de innovación: Un espacio para el desarrollo de las pymes, México Edit. Textual y UAM, 2009, pags. 33-80.

${ }^{9}$ Claudia de Fuentes (2009:136 y 137) presenta una tabla de variables empleadas por pymes para la construcción de las derramas de conocimiento de empresas grandes, en la que destaca la movilidad y capacitación del empleados de firmas globales y los tipos de vínculos técnicos y administrativos con los clientes. 
cionar a las temporalidades y espacialidades de las firmas globales ${ }^{10}$. Para poder analizar esto, un elemento adicional a tomar en cuenta, es la orquestación de un ambiente innovador en el espacio regional, en donde además de la relación interempresa, se genere una base institucional en el plano regional capaz de disparar procesos de industrialización endógenos, incorporando la colaboración y el apoyo de otros actores sociales, conformando así un sistema multi-focalizado de innovación (Castells y Hall, 2005). La generación de ambientes innovadores no son procesos de generación espontánea. Cada región tiene que demostrar su viabilidad y capacidad, en cuanto a disponibilidad de una infraestructura material y social para generar condiciones que hagan factible la instalación de filiales de firmas globales, la cual es una condición estratégica para generar las bases de un capital colaborativo (Fountain, 1997). Este último no se construye exclusivamente por la transmisión de conocimiento codificado sino que reconocen la importancia de la generación de condiciones para compartir experiencias, estimulando relaciones informales o lo que Asheim (2007) denomina "conocimiento pegajoso" que es lo que permite a las empresas internalizar externalidades.

\section{Metodología}

Las etnografías que pretenden hacer estudios sobre las cadenas de producción globalizadas se enfrentan al reto de seleccionar una estrategia que, desde lo local se pueda comprender su complejidad, en tanto muchas de las relaciones sociales no son presenciales, gran parte de la interacción se da de manera virtual e incluso son "invisibles" esto es, son incorporadas a la normativa y a las regulaciones de las cadenas. Para resolver este dilema, en esta investigación se optó por centrar la atención en las experiencias y percepciones de los actores sociales involucrados, así como de sus prácticas de comunicación cara a cara y a distancia. Para ello, se realizaron varias visitas a las filiales ubicadas en el país y a empresas nacionales, en las que se pudo observar la dinámica cotidiana de los grupos de trabajo, así como los mecanismos de comunicación con diversos agentes sociales tanto establecidos localmente como los múltiples contactos que se realizan a través de plataformas virtuales. El hacer trabajo de campo en empresas tiene serias limitantes. En las grandes firmas, el hermetismo y reserva reserva a la que se comprometen sus ejecutivos restringe el acceso a ciertas áreas y a información. Esto se subsana si existe alguna relación previa que ofrezca confianza de que no hay interés en difundir y aprovechar información técnica confidencial para fines comerciales ${ }^{11}$.

${ }^{10}$ David Harvey (2008) en su libro La Condición de la Posmodernidad utiliza los conceptos de infraestructura física y social para referirse a las dimensiones materiales y sociales del proceso de acumulación que no están directamente integradas al proceso productivo. Esto es, se refiere a todas las instituciones de respaldo que hacen posible que la maquinaria de la generación de valor camine. Esta infraestructura social, además de su papel central en la formación de capital humano, tienen el compromiso de generar actividades de impacto regional en cuando a la generación de conocimiento aplicado al desarrollo económico de las diferentes regiones.

${ }^{11}$ No obstante hay que cumplir con cierta normativa para poder ingresar, como previamente enviar la lista de preguntas, una carta oficial de la universidad que respalda el proyecto, no poder hablar con operarios en el lugar de trabajo por razones de seguridad del trabajador y ritmos 
El centrar el análisis en el actor permite apreciar las similitudes y diferencias en la manera de reaccionar a circunstancias estructurales particulares, poniendo hincapié en la producción de conocimiento a través de la interacción social y de la influencia de factores tanto del entorno inmediato como de fuerzas globales. Estos actores sociales presentan especificidades en la manera de movilizar recursos, construir su capital social o colaborativo para hacer frente a circunstancias que simultáneamente responden tanto a elementos locales y coyunturales como a situaciones influidas por procesos supra-locales que inciden en los márgenes de maniobra de sus empresas. Es así, como "las opciones individuales están influidas por marcos más amplios de significado y acción y por la distribución del poder y recursos en la arena más amplia" (Long, 2007: 44).

Dos fueron los actores sociales seleccionados: personal que ocupa algún puesto de mando en firmas globales instaladas en México, en particular de la industria automotriz en virtud de ser una de las industrias más dinámicas y más innovadoras que existen a nivel global. Asimismo, empresarios de pequeñas empresas productoras de moldes y troqueles. Se escogió esta especialización industrial porque son componentes indispensables en la fabricación de cualquier producto. Como se pudo apreciar en la nota a pie de página, número 4 , su manufactura ha exigido adecuaciones tecnológicas en concordancia con la reconversión industrial actual. Seleccionar esta especialización productiva de las empresas mexicanas fue importante para el propósito de este artículo pues exige de una estrecha comunicación con los clientes pues los moldes y troqueles son "hechos a la medida." Se trata de empresas intensivas en tecnología, en la que empresas mexicanas se posicionan de manera marginal en la demanda de firmas globales ubicadas en el país. Se reconoce que México es un importador neto de moldes. El International Trade Center (2011) ubica al país como el mayor importador de moldes de los Estados Unidos. En 2009 concentró el 9.6\% de estas importaciones. Estos datos ilustran que las empresas globales requieren moldes y troqueles de alta complejidad. Dicha demanda, por lo que muestran estas cifras, no es atendida por la planta productiva endógena. Esto resulta paradójico pues una de las fortalezas que atrajeron al capital norteamericano a instalar plantas de fabricación de motores a México, en la segunda mitad del siglo XX, fue el reconocimiento de una especialización en el país en el ramo metal-mecánico.

\section{Breve ubicación contextual}

Durante la década de 1990, México firmó múltiples acuerdos comerciales a nivel internacional. Esto último se ha concebido como respaldo a un modelo de desarrollo industrial que privilegia la apertura de fronteras, teniendo como disparador estratégico, la entrada de fuertes inversiones de capital extranjero que han instalado filiales con tecnología avanzada y con procesos organizativos altamente competitivos. Esto es resultado de la política industrial del país que apostó por atraer inversiones de

de productividad, no sacar fotografías, esperar a que los altos directivos acepten el ingreso a la planta, etc. Después de estos requisitos de entrada, la relación generalmente se relaja y da oportunidad de conocer más a fondo la problemática a estudiar. 
Europa, Asia y Estados Unidos para producir bienes que tienen como prioridad, su venta en los mercados mundiales más importantes, sobre todo, a los Estados Unidos. Este modelo de industrialización no sólo está controlado por el capital global, sino que además dirige el devenir del desarrollo industrial endógeno, incluyendo de manera selectiva, a las empresas nacionales que tienen la capacidad de responder a sus parámetros de productividad e innovación (Bueno, en prensa; Capdeville, 2005). Es en este contexto donde se desarrollan los casos que se presentarán a continuación.

\section{El caso de una armadora automotriz ${ }^{12}$}

General Motors Company ${ }^{13}$ instala en 1935, su primera planta en México, ahora cuenta con cuatro complejos industriales. En este ensayo se destaca el Centro Regional de Ingeniería (TREC) ubicado en el Ed. de México que es una unidad de diseño e investigación. Este centro forma parte de la red Global de Ingeniería y Manufactura de General Motors, comienza operaciones en 1995, contado hoy día, con más de 700 investigadores que están vinculados con otras unidades en todo el mundo. Llama la atención que este centro de ingeniería y diseño no se vincula con el entorno inmediato y por ello lamentablemente, la región donde se ubica no recibe ningún impacto. TREC se divide en áreas funcionales que se especializan en la ingeniería, prueba y validación de innovaciones. Todos los proyectos de este Centro Regional operan con equipos multinacionales y están vinculados directamente con las operaciones estratégicas de la corporación ${ }^{14}$.

La complejidad de esta automotriz es tal, que la información que se va a reportar en este artículo proviene de la experiencia de uno de los ingenieros que trabaja para TREC y que hoy día participa en un proyecto con GM Corea, como él afirma: "me revisan mis objetivos en México, pero mis resultados se dan en otras regiones del mundo". Es interesante la información que aporta porque permite comprender la dinámica en el plano global de la proveeduría de moldes y troqueles y la manera en que se insertan las filiales de México y Corea.

A continuación se presenta la estrategia de abastecimiento de moldes de la armadora a nivel global y posteriormente, se analizará las posibilidades de que proveedores mexicanos puedan abastecer de moldes. En un proceso de innovación disruptiva

\footnotetext{
${ }^{12}$ La información de las dos firmas globales fue recabada por Carmen Bueno y Homero Arriaga en Diciembre 2010.

${ }^{13}$ Hasta 2009 se llamaba General Motors Corporation, pero en 2009 se declara en quiebra y cambia su razón social.

${ }^{14}$ Uno de los ingenieros de este Centro comentó que su primer proyecto fue dar soluciones de ingeniería a un diseño alemán que se iba a producir en Estados Unidos. El se mantenía trabajando en Toluca pero con juntas virtuales semanalmente con los ingenieros de Estados Unidos y esporádicamente tenía que consultar a los ingenieros ubicados en Alemania para cuestiones técnicas y de legislaciones locales y federales. Para mantener la comunicación utilizaban todas las herramientas de las tecnologías de la información. Para evitar problemas emanados de la comunicación intercultural de estos equipos multinacionales, GM tiene cursos en línea para comprender la cultura del interlocutor. Para ello se contrata un paquete virtual que se llama Globesmart con el objetivo de evaluar las habilidades de sus ejecutivos de ser "ciudadano global" y saber interactuar en múltiples foros.
} 
o estratégica como la que se desarrolla en TREC, la selección del productor de moldes entra en el momento en que se realiza un análisis de factibilidad de la innovación. Este es el tramo inmediatamente anterior a que se toma la decisión de implementar todos los cambios de proceso, tecnología e insumos para lanzar al mercado un nuevo producto. Las firmas automotrices son propietarias de algunos de los moldes. Se trata de moldes críticos que los distinguen en el mercado. Para este tipo de moldes, las armadoras aportan las especificaciones del diseño y pagan los costos emanados de producción. Sin embargo, los proveedores de primera fila son los que monitorean y coordinan la relación con los molderos. Esto implica una interacción triangulada, que además requiere la orquestación con otras vinculaciones como son los proveedores de acero o del material que se va a utilizar y abastecedores de materia prima.

Hay también moldes de componentes específicos como sería el switch de arranque, que las armadoras compran como "caja negra". Esto quiere decir que ellos no intervienen en el diseño, ni en la producción, sino que son componentes estandarizados, en donde el proveedor también global, vende el mismo producto a diversas armadoras. Hay un tercer tipo de molde que el informante nombró como "paquete extra" que son los que elige libremente el proveedor de la armadora o alguna filial de ésta. Se trata de moldes que no son críticos, que no reportan un alto nivel de complejidad. Estos son precisamente los que abastecen pequeñas empresas locales como el caso que se presentarán en este artículo.

Después de describir la lógica de proveeduría de esta firma vemos que los lazos fuertes de proveeduría los tiene con firmas que operan con la misma lógica. Este hermetismo es un gran desafío para la planta industrial de capital nacional pues la brecha se ensancha cada vez más, entre el grupo selecto y la producción "periférica" que generalmente los ubica en el "paquete extra". Hay ocasiones en que una innovación cuyos costos han sido absorbidos por la empresa moldera, es rechazado por el área de finanzas de la armadora porque implica un alza de precio significativa que no están dispuestos a pagar. Esto implica que, a pesar de que las empresas de moldes están procurando ser competitivos y generar innovaciones, esto no garantiza que se va a firmar un contrato de proveeduría, a menos que consideren que posiciona a la armadora estratégicamente en el mercado.

Cuando se le preguntó al ingeniero de TREC, si tenía proveeduría local de moldes críticos, el comentó: "Sí, en la región de Norteamérica nuestros moldes se manufacturan generalmente en Estados Unidos, y los trasladan a sus plantas de proveedores de primera fila en San Luis Potosí o Querétaro o donde estén en México". Esto da cuenta de una perspectiva mundial de los empleados globales. La región está identificada como un espacio supranacional, respaldado por un acuerdo comercial (NAFTA) que se firmó en 1994 con Estados Unidos y Canadá y que permite mover maquinaria, herramentales sin restricciones arancelarias.

Uno de los ejemplos puntuales que comentó nuestro informante sobre la brecha de México con países industrializados es que no hay aceros especializados en 
México ${ }^{15}$, se tienen que importar. Una de las contradicciones de los tratados comerciales que ha firmado México es que la Secretaría. de Economía pone candados para la importación de bloques de acero. Sin embargo, abre las fronteras para importar moldes. Cuando los proveedores nacionales de moldes logran importar acero, éste tiene un costo superior al que pueden tener sus competidores en Estados Unidos o China, lo cual los sitúa en desventaja competitiva. Esta situación es diametralmente opuesta en el caso del Centro de Ingeniería de General Motors en Corea. En este país están dos grandes proveedoras de moldes críticos que tienen operaciones en Asia ${ }^{16}$. También China cuenta con recursos estratégicos para ser competitivo a nivel mundial, al punto que exportan moldes a todo el mundo. La desventaja competitiva con la producción mexicana es que China y Corea cuentan con siderúrgicas que producen aceros complejos, además de operar con economías de escala que les permite proveer a su mercado interno y expandir su presencia mundialmente. Estos recursos son la base para que estos países asiáticos puedan realizar fuertes inversiones en tecnología e innovación, además de un capital humano capacitado y barato.

\section{El caso de una abastecedora mundial de componentes automotrices}

A inicios de la década de 1980 se funda Elastómeros Halcón, empresa mexicana para la producción de piezas de hule para la industria automotriz. El origen de esta empresa mexicana es producto del decreto presidencial que exigía que hubiese un $60 \%$ de autopartes producidas en el país para surtir a las ensambladoras. En la siguiente década, ante la firma de los acuerdos comerciales se asocia con una empresa estadounidense que después es comprada por una empresa sueca.

$\mathrm{Su}$ actividad principal en México es producir partes de hule para la eliminación de vibraciones de chasis para prácticamente todas las armadoras de la industria automotriz. Esta autoparte, como muchas otras, se ha complejizado muchísimo. Ahora son diseños de ingeniería que requieren de varios cientos de mezclas de hule. La planta de México está directamente relacionada con las oficinas en Estados Unidos. Es allá donde se seleccionan todos los proveedores, incluyendo sus abastecedores de moldes y troqueles.

En México proveen a Nissan, Volkswagen y Ford. Ninguno de sus clientes se encuentra en la zona industrial próxima a donde está instalada. Además, esta planta exporta la mayor parte de su producción a Estados Unidos, Canadá y Brasil. Esto da cuenta de que la proximidad no es un indicador importante para el abastecimiento de estas piezas. Su materia prima es natural, no la suple por ningún material sintético pues no tendría la resistencia que se necesita. El hule que utilizan en la planta de México proviene de Tailandia. Otro de los elementos que constata la estrategia desterritorializada de las firmas globales es la importación de un producto que se podría obtener en México pues el hule crece en las zonas tropicales del país y en este

${ }^{15}$ Nos comenta que el importador más cercano a México es ArcelorMittal que es una siderúrgica cuya tecnología es propietaria de aceros especializados para la industria basada en tecnología.

${ }^{16}$ Estas dos empresas son Arrk y Shenyang Moca Auto Mold. 
sentido, la firma global no genera ningún compromiso con el desarrollo regional de los lugares donde se ubica.

Cada pieza que Trelleborg produce, requiere de varios moldes, pueden ser dos, a veces cinco. Cada molde requiere varias placas que se interconectan y que tienen incorporada diferente tecnología. Estos moldes son propiedad de la armadora y esta última es la que absorbe los gastos de su manufactura. No obstante es responsabilidad de Trelleborg coordinarse tecnológicamente con la productora de moldes e invertir en la maquinaria donde se instalarán estos moldes. La inversión en tecnología es constante y necesaria para poder ofrecer productos de alta calidad e innovadores a nivel mundial.

Es importante que las unidades de esta firma donde se generan las innovaciones estratégicas, estén próximas a los centros de diseño de las armadoras de automóviles. Con ellos mantienen una permanente comunicación, además de que la proximidad facilita los encuentros para los nuevos productos que van a salir en los próximos años. Esto constata que el concepto de medio innovador de Castells y Hall (2005) responde a un modelo hegemónico de industrialización y pierde alcance explicativo cuando se analizan nodos de estas cadenas de producción ubicados en la periferia, como son los casos aquí estudiados.

Los moldes que se usan en la planta de México provienen de proveedores ubicados en Michigan, Estados Unidos, con quienes se ha fomentado una larga relación de 20 o 30 años. Estos han acumulado importante conocimiento en la producción de moldes complejos para el vaciado del hule a lo largo del tiempo, lo cual genera un importante vínculo basado en la confianza y en la certeza de que la comunicación de lo que se demanda y lo que se ofrece es asertiva. Como afirma el Gerente: "Los productores de moldes se les pide estar certificados en ISOTS que es la acreditadora mundial para la industria automotriz, pero lo más importante es que saben los secretos del hule". Esto da cuenta que el conocimiento tácito sigue ponderándose de manera importante en la manufactura basada en innovación estratégica.

A pesar de tener proveedores de moldes que han probado su expertise a través del tiempo, la armadora siempre exige que se busquen nuevos proveedores que puedan ofrecer un mejor precio. La planta en México tiene que dar respuesta a la política de la firma de buscar proveedores que permitan la reducción de costos. La alternativa más viable encontrada por la planta de Trelleborg en México fue buscar productores de moldes en China, a pesar de la distancia. El gerente considera que hay una serie de obstáculos para comenzar un proyecto de desarrollo de proveedores de moldes en México y los moldes chinos han probado ser baratos y de muy buena calidad.

En cambio la percepción que el gerente de Trelleborg tiene sobre las empresas locales productoras de moldes es que uno de sus principales obstáculos es competir con empresas que no sólo cuentan con mayor infraestructura, sino con conocimiento acumulado y con una relación de confianza afianzada a través del tiempo. Además estas empresas mexicanas tienen que pasar por una serie de filtros, el más importante es la inspección directa del Centro de diseño ubicado en Estados Unidos. Entre los requisitos está el tener la certificación de calidad internacional ISO que no muchos lo tienen, pues les implica un costo alto en relación a sus ingresos. Les exigen 
también una altísima precisión en cuanto a especificaciones, lo que implica tener la tecnología y un modelo organizativo con parámetros globales que en la mayoría de los casos está ausente. Otros desafíos que tiene que enfrentar la proveeduría local es tener la suficiente solvencia financiera para poder invertir en tecnología y superar la "cultura de ferretería, de vivir de la oferta-demanda diaria", como lo mencionó nuestro informante. La liquidez de la empresa es importante para poder afrontar las fluctuaciones de los insumos como el acero, en contratos de cinco años. Esta proveeduría local, además se topa con otro obstáculo que es el no estar próximo a los Centros de Diseño.

No obstante todas estas limitaciones, el gerente general, ante las presiones de las armadoras de conseguir fabricantes de moldes que fuesen más económicos, propuso un programa de desarrollo de proveedores en México, apoyándose en diversos programas de gobierno y en las cámaras industriales. Para ello tenía que colaborar con distribuidores de matera prima en Estados Unidos, por las especificidades que tiene el diseñar un molde para inyección de hule. La condición que pusieron estos distribuidores era ofrecer capacitación a 10 proveedores. A esta convocatoria sólo acudieron tres empresas. Estos datos dejan ver dos cosas: que la proveeduría de moldes para firmas globales implica decisiones y colaboraciones multinivel al interior de la firma y con diversos proveedores de insumos, incluso de maquinaria que, como en este caso, no son firmas que se encuentran en el país y que imponen ciertas condiciones para poder entablar relaciones de largo alcance y donde se da un fuerte componente de generación de conocimiento. Lo segundo es que, hay un sinnúmero de empresas de moldes con muy baja tecnología y son pocas las que pueden reaccionar a la demanda de firmas globales. Estas últimas, además no les interesa el desarrollo de proveedores en regiones donde la planta industrial, no cuenta con la base tecnológica, organizativa y financiera para dar certidumbre de colaboración.

Adicional a esto, en México hay una total ausencia de programas de formación a nivel profesional sobre el manejo de hule, a diferencia del plástico o de uno de sus derivados que es el pet. ${ }^{17} \mathrm{y}$ también hay carencias en la profesionalización en el sistema de educación superior de moldes complejos en México. Esta carencia en externalidades que apoyen la generación de capital humano para esta rama manufacturera se tiene que suplir con formación en la práctica directamente en los lugares de trabajo y en cursos que ofrecen directamente estas grandes firmas, incluso hay filiales que instalan escuelas técnicas al interior de sus plantas. La crítica sobre la falta de capital humano capacitado es recurrente y refleja un sistema educativo deficiente y a la saga de los requerimientos de la planta industrial global ubicada en el país y por otro lado, el incesante desarrollo de innovación en las firmas globales que está provocando una sobre-especialización en actividades específicas, basadas en conocimiento y alta tecnología.

${ }^{17}$ Las universidades que se especializan en la formación en el manejo del hule se encuentran cerca de la industria llantera. En el caso de Estados Unidos están en Ohio y Michigan. 


\section{Una empresa mexicana abastecedora de moldes para firmas globales}

A continuación se presentan la trayectoria de una empresa familiar de capital mexicano que ha podido superar las limitantes de la mayoría de las empresas que producen moldes y troqueles en el país. Se analiza ¿qué es lo que la distingue en cuanto a: visión empresarial, recursos, estrategias de vinculación y habilidades para poder evolucionar organizativa y tecnológicamente y así reaccionar positivamente a la demanda de firmas globales ubicadas en el país ${ }^{18}$. Se trata de una empresa que ha podido ingresar a la zona de frontera (Sassen, 2001) porque tuvo los recursos y capacidades internas y de vinculación para sustituir troqueles y moldes que se importaban del extranjero. Si tomamos en cuenta los parámetros de la producción global, los componentes que esta empresa produce no están aún considerados como productos críticos de alta complejidad, pero si se compara con la generalidad de empresas de esta rama productiva en el país, sí destaca por reportar actividades generadoras de nuevo conocimiento y por tanto tener posibilidad de innovar bajo parámetros internacionales.

Esta empresa familiar se ubica cerca del cluster automotriz de Volkswagen, en este artículo se denominará "la Poblana". El director general se formó profesionalmente en ingeniería mecánica en una universidad privada en Puebla, filial de una red de universidades estadounidenses. Posteriormente, recibe una beca del gobierno mexicano para estudiar una maestría en Ingeniería Industrial en Inglaterra. Su trayectoria empresarial comienza en el 2001, cuando abre una empresa representante de herramientas de corte de madera de origen alemán. Esta empresa cierra a los pocos meses de haber arrancado porque el especialización productiva de producción de muebles de madera estaba en declive. Posteriormente decide junto con sus hermanos buscar oportunidades de proveer algún servicio a la industria automotriz y sin tener experiencia previa en el sector metal-mecánico ofrecen el servicio de reparación de troqueles. Durante ese periodo identificaron un nicho de oportunidad, ofreciendo un componente que incorporara alta tecnología y que no se ofertaba en el mercado nacional. Es así como en 2006, optaron por fabricar dispositivos de control para piezas manufacturadas con moldes o troqueles ${ }^{19}$. Su primer cliente fue una empresa de autoparte de primera fila ubicada en el parque industrial FINSA, donde se encuentran todas las proveedoras de la Volkswagen. En un lapso muy corto, ha logrado obtener reputación en el medio, por lo cual producen estos componentes para otras armadoras y sus proveedores en otros estados del país.

En la trayectoria se puede apreciar que el respaldo y colaboración familiar sigue siendo muy importante. Llama la atención el corto ciclo de aprendizaje de su dueño para posicionarse en un mercado que desconocía hasta hace un lustro, respaldándose fuertemente en su educación formal en México y en el extranjero. Esto da cuenta de la implementación combinada de los diversos mecanismos de derrama

${ }^{18}$ En este ensayo se guardará el anonimato de las empresas, los empresarios, empleados de estas empresas, otorgándole el nombre de la Poblana.

${ }^{19}$ La información de campo de esta empresa fue recogida por los asistentes de investigación: Rodrigo Ramírez, Joanna Felix, Homero Arriaga y Enrique Cornejo en el primer semestre del 2011. 
de conocimiento apropiados por los dirigentes de estas empresas y propuestos en el texto de Gabriela Dutrénit y Claudia de Fuentes (2009).

La empresa Poblana básicamente centra la oportunidad de proveer a la industria automotriz en la adquisición de tecnología de clase mundial de origen europeo o norteamericano. Los proveedores de maquinaria les otorgan crédito y les dan capacitación. Esta combinación de productos-capacitación-servicio de soporte es central. $\mathrm{Su}$ estrategia es capacitar directamente con los proveedores de tecnología a los jefes de sección que a su vez, enseñan en la práctica a los operarios, en una suerte de capacitación en cascada. La centralidad en la tecnología genera una de las tensiones principales en esta empresa pues no siempre es factible invertir en maquinaria de última generación, pues aún no aseguran una demanda sostenida en cantidad como para arriesgar en invertir en tecnología que puede estar subutilizada. En sustitución, una de las estrategias es optimizar la maquinaria con la que cuentan. Esto de alguna manera, genera una importante acumulación de procesos de aprendizaje al interior de la empresa, sin embargo, los pone en desventaja en cuanto a calidad y tiempo de entrega y por tanto se ve limitado a reaccionar a las temporalidades (Sassen, 2001) de la dinámica global, que exige ritmos de entrega que sólo son posibles si se cuenta con la tecnología y los insumos adecuados. También en la operación de esta empresa hacen mucho hincapié en invertir en instrumentos que optimicen la comunicación entre los diferentes departamentos y con sus clientes. El compartir información, sincronizando tiempos y actividades es una de las fortalezas de esta empresa y elemento de confianza con sus clientes.

En cuanto a la calidad de sus recursos humanos, la Poblana como se comentó anteriormente, implementa un aprendizaje en cascada, donde se privilegia la especialización en las diversas actividades de esta empresa, a la vez que se aplican prácticas de aprendizaje por interacción (learning by interacting). Esto se complementa con el almacenamiento de conocimiento codificado en una biblioteca virtual que les permite optimizar el conocimiento acumulado por la empresa y no sólo el incorporado en la experiencia directa del personal. La importancia otorgada a la inversión en maquinaria y el método interno en cuanto a derrama de conocimiento, así como el acceso y manejo de información de procesos vigentes y experiencias pasadas al interior de la empresa, es una importante fortaleza para potenciar sus capacidades internas y reaccionar con mayor prontitud a la demanda de firmas globales. En estas empresas se produce un conocimiento pegajoso (Asheim, 2007) fomentando un ambiente innovador a su interior, que es estratégico para poder reaccionar a los parámetros de calidad de las firmas globales.

El ser proveedores de firmas globales es visto como una gran oportunidad porque les da prestigio, además como se pudo apreciar anteriormente, los somete a fuertes exigencias de aprendizaje interno para cumplir con los parámetros de calidad, precio y tiempo de entrega de los circuitos de producción globales. A continuación se presenta la forma que toma esta vinculación, las presiones y la posibilidad de que en esta relación, se de algún tipo de derrama de conocimiento para generar procesos orientados a la innovación. 
Esta empresa reconoce que su posibilidad de abastecer a empresas globales depende en gran medida de que estas últimas busquen abastecerse localmente, bajo la práctica de sustitución de importaciones de algunos componentes, con la finalidad de reducir costos de operación. Otra estrategia es ampliar los servicios que provee, algunos son sólo de reparación y mantenimiento de moldes que combinan con los de alta complejidad. Los primeros sostienen el flujo de recursos, mientras que los segundos, le permite generar capacidades internas e incrementar su conocimiento innovador. Esto es lo que ha llevado a esta empresa a alcanzar prestigio y reconocimiento en su especialidad. Para esta empresa las certificaciones son una carta credencial obligada, como el ISOTS. La opinión de los dueños de esta empresa es que facilita la comunicación con los clientes pues estandariza los criterios de calidad, les induce a mantener prácticas operativas que les ayudan a mejorar su productividad y monitoreo de los procesos. Esto se ha convertido en un instrumento de codificación estandarizada de expectativas de ambas partes. Además, tiene el efecto de generar esquemas compartidos de comunicación con la proveeduría global, una suerte de lingua franca que genera certidumbre y la apropiación de una cultura de calidad bajo parámetros estandarizados globalmente.

La utilización de medios informáticos para la comunicación con sus clientes también ha sido un elemento que le permite un acercamiento y mejor entendimiento de expectativas referidas a calidad, manejo de insumos, etc. Además, la empresa Poblana procura realizar visitas constantes para afianzar las relaciones cara a cara en el seguimiento de los proyectos. La comunicación constante, conlleva procesos de aprendizaje, de compartir información tácita, de comprender las necesidades y la cultura operativa del cliente. No obstante, la vinculación con las firmas globales de primera fila es aún de corte instrumental. Aquí hay que hacer hincapié que son filiales de partes y componentes que tienen que alinearse a las exigencias de las armadoras y que su margen de maniobra en lo local es bastante reducido. Además en las etapas iniciales de vinculación entre empresa nacional-firma global, hay un fuerte componente de información codificada, que si bien perfila aún, una relación más bien distante, ésta puede sentar las bases para evolucionar a una relación de mayor confianza.

Las tensiones que recurrentemente se presentan en la vinculación con firmas globales según argumenta uno de los técnicos de la empresa Poblana es que, los clientes los presionan demasiado por los tiempos de entrega y estos últimos no toman en cuenta que parte de los insumos que necesitan son importados y los tiempos para su abastecimiento no corresponden con las exigencias de tiempo de la proveeduría global. Esta situación hace que pueda haber un retraso, lo que obstruye la posibilidad de avanzar a relaciones más estrechas con sus clientes. Otra limitante es que los clientes globales también les exigen mantener precios estables, independientemente de las fluctuaciones del precio del acero y de su dependencia de tecnología del exterior, pues todos los recursos tecnológicos son importados. La negociación de pago es un gran problema para mantener el flujo de recursos, esto llega a trastocar la planeación de la producción. Otra queja de la relación es que las firmas globales suelen minimizar el valor de la innovación de un dispositivo, no obstante que, desde 
la perspectiva de las empresas locales, cualquier modificación, les significa un rediseño de herramentales, adecuaciones a los procesos de producción, mayor capacitación de operarios, incluso inversión en nueva maquinaria. Los costos implícitos en esto, son obstáculos que afirman reiteradamente, los pone en desventaja en relación a su competencia internacional.

Por último, la importación de moldes del extranjero por parte de la industria automotriz es visto por la empresa Poblana como una ventaja que han sabido aprovechar, no sólo para ampliar su mercado, ofreciendo sus servicios de reparación y mantenimiento sino que se han apropiado de información tecnológica contenida en estos componentes, realizando una suerte de "ingeniería a la inversa". Estos servicios de mantenimiento y reparación les han servido para abrir puertas y darse a conocer en cuando a su capacidad tecnológica y organizativa. El análisis de este caso da cuenta de la generación de estrategias de empresas locales para sacar ventaja de las vinculación con firmas globales, aunque aún sean de corte instrumental, superando todas las restricciones de una relación en la que persisten aún asimetrías, pero que muestra condiciones para evolucionar positivamente, esperando en un futuro afianzar su posición en la red de proveeduría global para participar en procesos de generación de conocimiento de mayor complejidad.

Las oportunidades que se han abierto para empresas mexicanas como la Poblana están centradas en la estrategia de las firmas globales instaladas en el país de sustituir importaciones, ya no por políticas proteccionistas implementadas por el Estado, como sucedió antes de la década de 1980, sino por una lógica de mercado orientado a buscar proveedores que les puedan ofrecer la misma calidad pero a menor costo. Los recursos limitados en los que operan las empresas nacionales se suplen por el uso óptimo de sus capacidades dinámicas que les permiten generar competencias funcionales para reaccionar a los desafíos de los parámetros de la producción global y de esta manera acceder a las zonas de frontera a las que alude Sassen (2001).

A diferencia de las firmas globales, las pequeñas empresas locales requieren echar mano de múltiples relaciones en el entorno inmediato, además de las oportunidades de aprendizaje y colaboración en el espacio construido por la relación proveedor-cliente. Hay otras vinculaciones donde además de que fluyan recursos tangibles en apoyo a la producción, se comparte información y saberes que permiten generar conocimiento orientado a soluciones específicas o bien a actualizarse en las mejores prácticas. En este sentido, esta empresa busca continuamente apoyos a través de la relación con diversos agentes: otras empresas del mismo especialización productiva, los proveedores de materia prima y maquinaria, las instituciones de educación media y superior, asociaciones, ferias y por último, la relación más frágil, las instancias de gobierno en apoyo al sector productivo. La pregunta que se desprende es: ¿Están estas vinculaciones respaldando un ambiente colaborativo orientado a generar nuevo conocimiento?

Se pudo constatar que las relaciones horizontales con empresas de la misma especialización productiva giro es una constante. Se trata de una subcontratación reactiva, circunstancial de apoyo ante contingencias y/o momentos coyunturales. Es una manera de no desaprovechar las oportunidades de demanda de sus componentes, 
al mismo tiempo que permite expandir su cartera de clientes, lo que ha sido una estrategia muy exitosa para contar con el flujo de recursos que necesita para su operación. Esto ha sido un paliativo para no tener que incrementar sus inversiones en maquinaria, ni en capacitación. Este cálculo empresarial redunda en el control de riesgos financieros que podrían someterlos a crisis que deteriorarían la evolución de la pequeña empresa que quiere abastecer a los circuitos globales. Aquí lo que se puede apreciar es que en este tipo de colaboraciones, hay la posibilidad de compartir conocimiento tácito, pidiendo "consejos o tips" para resolver problemas en la inmediatez. Estos apoyos generan un ambiente donde se comparten diversos recursos en relaciones de equidad, donde impera el respaldo mutuo: "Hoy por mí, mañana por ti" ${ }^{20}$. Esto que es algo implícito en estas colaboraciones resulta de gran valor porque son relaciones basadas en la confianza mutua y en la oportunidad de compartir recursos de diversa índole.

Otra alternativa de colaboración es la transmisión de información y capacitación formal e informal por parte de los proveedores de maquinaria y de materia prima. Esto suple el hermetismo de las firmas globales para transmitir información "confidencial" que es accesible sólo cuando se participa a sus programas de desarrollo de proveedores. De lo contrario, como es el caso de la empresa Poblana, las relaciones con las firmas globales son de corte instrumental. Los vínculos con sus proveedores generan efectos positivos en cuanto a la generación de prácticas colaborativas, se transmite información táctica derivada de la experiencia y del respaldo de información codificada incorporada a manuales. Estas son fuentes muy valiosas que contribuyen a la profesionalización de las pequeñas empresas mexicanas. Esto lo complementan con otros canales de acceso a información como las páginas comerciales por internet o a través de revistas especializadas en la producción de plásticos o en metal-mecánica que son complemento obligado del auto-aprendizaje en la práctica cotidiana. Otro canal al que acceden para obtener información actualizada es la asistencia a ferias nacionales e internacionales para actualizar sus conocimientos, comprar tecnología, así como conocer y compartir experiencias con otras personas que están en la misma especialización industrial.

La colaboración con universidades, tecnológicos o escuelas técnicas para generar procesos de generación de conocimiento está ausente. Tampoco estas empresas han acudido a los Centros de Investigación Técnica del gobierno para buscar asesoría técnica. El único rol que le atribuyen a los centros de enseñanza es el proveer al mercado de trabajo, capital humano especializado en la producción metal-mecánica. En este sentido manifestaron diversas inconformidades que se suplen al contratar a quienes pasaron por los centros de capacitación que establecen algunas firmas globales. En el caso de Puebla, hay varias empresas del cluster de la Volkswagen que por las deficiencias de encontrar personal capacitado en la región, han abierto centros de enseñanza práctica. El personal que estas firmas no contratan directamente, están disponibles para otras empresas y firmas de la región, siendo una alternativa que ha tenido un impacto positivo de derrama de habilidades por parte de las firmas globales.

${ }^{20}$ Este es un dicho muy utilizado en México que demuestra las prácticas de reciprocidad. 
Sin embargo, se puede constatar un importante liderazgo de los dueños de estas empresas para fomentar vínculos con diversas externalidades. Por su parte, el director de la empresa Poblana participa en Canacintra porque es una manera de relacionarse y obtener información de primera mano sobre la situación legal, contable y de seguridad social para los trabajadores. También buscó apoyo de una empresa de consultoría de negocios llamada CRECE. A pesar de que no logró obtener un crédito acorde a sus necesidades, sí aprendió instrumentos que le ayudaron en la administración de su empresa, como hacer un plan de negocios, saber medir riesgos financieros, así como llenar formatos para conseguir créditos. Estos programas que desarrolla el gobierno con la banca de desarrollo, muchas veces quedan truncos o bien quieren abarcar un espectro tan amplio de pequeñas empresas que terminan por apoyar a las más intensivas en trabajo que no requieren inversiones significativas en tecnología. Todo esto demuestra que aquellas empresas que han podido sobresalir de la precariedad en la que operan la mayoría de las pequeñas empresas nacionales, tiene un interés genuino por apoyar proyectos que coadyuven a generar un capital asociativo en el entorno inmediato.

No obstante, los pequeños empresarios reiteradamente mencionan que el gobierno debería de desarrollar política pública que deliberadamente apoye a las pequeñas empresas intensivas en tecnología. Una de las debilidades de esta vinculación es que los programas de gobierno están atados a los tiempos políticos. La empresa Poblana ha buscado acceder a otros programas, como por ejemplo INNOVA de CONACyT, que apoya al desarrollo de prototipos, patentes, para obtención de software pero no otorga suficientes recursos para la compra de maquinaria, que es uno de los recursos estratégicos más difíciles de obtener, por la inversión que esto implica. También esta empresa fue elegida para participar en un proyecto del estado de Puebla junto con el Instituto Poblano para la competitividad titulado 150 PYMES. Este procuraba la colaboración entre universidades y sector productivo. Dicho proyecto quedó trunco durante el cambio de gobernador y de partido político en el gobierno. Estos son ejemplos que ilustran los obstáculos que se interponen para que el gobierno sea un promotor importante del desarrollo regional, sobre todo para procurar condiciones para que las pequeñas empresas nacionales puedan operar y reaccionar a los parámetros de un modelo industrial liderado por firmas globales.

En este apartado se pudo constatar que las vinculaciones con el entorno inmediato son endebles como para reflejar la generación de un ambiente innovador con las características propuestas por Castells y Hall (2005). Las vinculaciones que han sido importantes para producir un capital colaborativo, son otras empresas de la misma especialización productiva, donde hay un interés mutuo aunque reactivo de apoyar en situaciones coyunturales. También las asociaciones ofrecen información de diversa índole y cursos de capacitación. Además de ser instancias de contacto donde conocen otros empresarios dedicados a la misma rama industrial. Sin embargo, éstas son sólo agencias vinculantes cuya fortaleza es propiciar encuentros que pueden o no derivar en oportunidades de colaboración para la innovación. Mientras que la infraestructura educativa se ha limitado a proveer al mercado de trabajo técnicos y profesionales que no respaldan su título con una educación que les 
permita desarrollar habilidades orientadas a generar nuevo conocimiento. También está ausente en la experiencia de este caso, proyectos de vinculación para la innovación con las instituciones de educación media y alta y con diversas dependencias de gobierno. Todo esto genera redes regionales poco coadyuvantes a la generación de procesos innovadores.

No obstante, se reporta un impacto más positivo en vinculaciones de índole supra-regional, por ejemplo, los proveedores de tecnología y materia prima que no siempre tienen representaciones en el entorno inmediato, ofrecen información y apoyo formal e informal, generando sinergias donde se transmite conocimiento que es considerado estratégico para la operación de estas empresas. También las ferias son espacios donde compran tecnología, además de propiciar encuentros informales donde se comparten experiencias de otras latitudes y que son altamente valoradas y en ocasiones apropiadas para la solución de problemas en la operación de las empresas. Es un conocimiento que se capitaliza al interior de la empresa y que a través de las vinculaciones informales propicia de manera circunstancial la derrama de conocimiento en el plano regional/local.

\section{A manera de reflexión}

Al analizar procesos globales en lo local a partir de la perspectiva del actor social, se abre un prisma en la comprensión de los complejos procesos y relaciones multinivel en las cadenas de producción globalizadas. La estrategia metodológica fue seleccionar tres estudios de caso que guardaran posiciones diferentes de la cadena de proveeduría global. Esto dio cuenta de prácticas y percepciones que cruzan y le dan identidad a la lógica de la producción global y otras que explican las asimetrías y desigualdades entre las posiciones dominantes y periféricas de los procesos de manufactura a nivel global.

Esta complejidad parte del hecho de que la firma global ha configurado un sistema autocontenido que despliega una infraestructura material y social para tejer de manera selectiva puentes con proveedores que han pasado por múltiples mecanismos de evaluación y prueba para acceder al inner circle. Se pudo apreciar que hay interacciones trianguladas, como el hecho de que la armadora decide y paga los moldes pero su proveedora es la que tiene que negociar con el productor de moldes y los abastecedores de materia prima. Esto implica que las decisiones y las acciones se dan en un espacio en donde confluyen diversos niveles de control y toma de decisiones y que exige de la orquestación de diversos actores sociales. Fue reiterativa la importancia de las relaciones de confianza de largo aliento que generalmente tienen lugar en los nodos centrales de las cadenas de producción. Es esta confianza la que permite que el conocimiento tácito fluya sin candados y permita una comprensión más integral del conocimiento codificado incorporado a la tecnología, a los insumos y registrado en bitácoras que llevan el seguimiento de una innovación. Para lograr mayor sinergia y eficiencia, el conocimiento codificado fluye y se comparte en sofisticadas plataformas cibernéticas. El poder acceder a estar plataformas implica consultar y generar información que es sumamente valorada y protegida por su alto contenido tecnológico. 
Dicho sistema es escasamente poroso, abre algunos resquicios para activar oportunidades para las pequeñas empresas nacionales. Dichas vinculaciones se pueden definir como periféricas dentro de la estrategia de innovación de las cadenas de producción desterritorializadas. En estas vinculaciones permean relaciones asimétricas, que devalúan los múltiples esfuerzos de las empresas locales para aportar productos innovadores. Por un lado, se les exige invertir en tecnología y en capacitación bajo estándares internacionales, al tiempo que cuando se trata de que las innovaciones incorporadas se reflejen en los precios de sus productos, las firmas globales minimizan estos esfuerzos afirmando que son simplemente ajustes. Esta ambigüedad es producto de posiciones jerárquicas disímiles que genera diversas tensiones.

Es por ello que la participación rectora de las firmas globales para la derrama de conocimiento a nivel regional/local es muy limitada. En esta investigación no se pudieron percibir proyectos que deliberadamente tejieran vínculos estratégicos con el entorno inmediato, por múltiples razones: la lógica misma de la firma global que ubica a las innovaciones disruptivas en los nodos centrales, donde efectivamente se llevan a cabo una densidad de vinculaciones estratégicas intra e inter firma y con diversas instituciones. La fuerte política de reserva que inhibe la posibilidad de compartir conocimiento privilegiado fuera del inner circle ante la amenaza de la piratería tecnológica. Los múltiples requisitos que exigen a sus proveedores para colaborar en proyectos de innovación, lo que implica que estas empresas demuestren desarrollar o bien contar con recursos financieros, tecnológicos y humanos para poder responder a las economías de escala y control sobre los riesgos que demandan los procesos orientados a la innovación.

Los únicos datos que podrían ser testimonio de derrama de conocimiento de las firmas globales a las regiones donde se ubican son: la información codificada y selectiva que brindan a sus proveedores locales, lo cual es apropiado e incorporado a los procesos internos de estas empresas como es el caso de la empresa Poblana y las escuelas de capacitación que suplen la deficiente capacitación técnica del sistema educativo del país. Sin embargo, la presencia de firmas globales sí ha activado de manera indirecta procesos generadores de conocimiento. Aquí se dio testimonio de que algunas empresas nacionales han podido ubicarse en las zonas de frontera de la globalización, echando mano de todos los recursos a su alcance para abastecer de componentes basados en tecnología a las filiales de las firmas globales ubicadas en el país. Desde la perspectiva de estas empresas se trata de acciones estratégicas, mientras que desde el punto de vista de las firmas globales forman parte del "paquete extra". Esto es, son innovaciones reactivas que responden a la necesidad de las firmas globales de sustituir importaciones para reducir costos, bajo la modalidad de tecnología transferida. Entre los recursos incorporados están las capacidades dinámicas al interior de las empresas. Estas capacidades se han convertido en una de sus fortalezas para suplir la falta de tecnología y financiamiento; también las múltiples colaboraciones y el respaldo ante contingencias u oportunidades coyunturales entre empresas del mismo ramo. Así como relaciones generalmente de tipo informal que ocurren en encuentros, como las ferias y el soporte de información tácita y codificada que les aportan proveedores de insumos, de tecnología y cámaras industriales 
que operan a nivel local. De algún modo se pude afirmar que se ha generado un "ambiente innovador a la mexicana" por todos los recursos tangibles e intangibles que de manera improvisada, poco sistemática se comparten en un entorno de mayor horizontalidad, generando un capital colaborativo donde fluye "conocimiento pegajoso", aunque este último no sea estratégico bajo los estándares globales.

La posibilidad de generar un ambiente innovador del tipo que se desarrolla en las tecnópolis estudiadas por Castells y Hall (2005) requeriría de que el gobierno replanteara su modelo de desarrollo industrial en beneficio de la planta industrial endógena y no sólo de desarrollar políticas públicas orientadas a atraer capitales extranjeros ${ }^{21}$. Aquí se pudo constatar la ausencia de las instituciones públicas de educación, los centros de investigación y otras dependencias de gobierno que tienen programas en apoyo a las pequeñas empresas para apoyar al desarrollo de la productividad y la activación de procesos generadores de conocimiento tecnológico. El Estado, en países como México, tiene que tener una posición estratégica para que empresas locales puedan ser competitivas y superar su condición de exclusión o marginalidad de los circuitos dominantes de producción y de generación de innovaciones.

\section{Referencias bibliográfícas}

APPADURAI, Arjun. (Ed.)

2003 [1996] Modernity at Large. Mineapolis: University of Minnesota

ASHEIM, Bjorn

2007 "Differentiated Knowledge Bases and Varieties of Regional Innovation Systems". Revista Innovation, vol. 20, no. 3: 223-241.

BATTEAU, Allen

2010 Technology and Culture. Illinois: Waveland Press.

BILBAO, Elena

2010 El poder global en la industria automotriz. México: Universidad Iberoamericana

BOURDIEU, Pierre

1995 Respuestas por una antropología reflexiva. México: Editorial Grijalbo.

BUENO, Carmen

en prensa "Lo que la Revolución no contempló en su agenda: El desarrollo tecnológico en manos extranjeras". Conmemoración del Centenario de la Revolución. México: Edit. Universidad Iberoamericana.

CASALET, Mónica. (Coord.)

2002 El desarrollo de la capacidad innovadora de las empresas: el papel del ambiente en la formación y consolidación de las capacidades tecnológicas. México: FLACSO.

${ }^{21}$ Corea del Sur y China en un lapso muy corto han podido generar un medio innovador competitivo mundialmente con el apoyo de sus gobiernos. 
CASAS, Rosalba; LUNA, Matilde (Coords.)

2000 Gobierno, academia y empresas en México: Hacia una configuración de relaciones. México: IIS, UNAM, Plaza y Valdés.

CASTELLS, Manuel; HALL, Peter

2005 Tecnópolis del mundo. La formación de los complejos industriales del siglo XXI. Madrid: Editorial Alianza

DUTRÉNIT, Gabriela; FUENTES, Claudia

2009 "Derramas de conocimiento y capacidades de absorción" en Sistemas regionales de innovación: Un espacio para el desarrollo de las pymes. México Edit. Textual y UAM, 33- 80 .

EDQUIST, Charles

2004 "Systems of Innovation:Perspectives and Challenges" en Oxford Handbook of Innovation.

FEATHERSTONE, Mike. (Ed.)

1997 Undoing culture. Globalization, postmodernism and identity. London: Sage Publications.

FOUNTAIN, Jane E.

1997 "Social capital: A key Enabler of innovation in Science and Technology" en Investing in Innovation, Lewis Branscomb and James Keller ed. Cambridge, Ma.: MIT Press.

HANNERZ, Ulf.

1996 Transnational connections. London and New York: Routledge.

HARVEY, David

2008 La condición de la Posmodernidad. Argentina: Amorrortu Editores

LEYDESDORFF, H.; ETZKOWITZ, H.

1996 http://investigacion.universia.es/spin-off/triple-helice/index.htm

LONG, Norman

1996 "Globalización y localización: nuevos retos para la investigación rural”. La sociedad rural mexicana frente al nuevo milenio, México: INAH, UAM, UNAM, Plaza y Valdés.

2007 Sociología del desarrollo: una perspectiva centrada en el actor, México: El Colegio de San Luis y CIESAS.

LUNDVALL, B, (Ed.)

1992 National systems of innovation: Towards a Theory of Innovation and Interactive Learning, London: Pinter Edit.

ROBERTSON, Ronald

2003 "Glocalización: Tiempo-Espacio y homogeneidad-Heterogeneidad". Juan Carlos Monedero (Ed). Cansancio del Leviatán. Problemas políticos en la mundialización. Madrid: Trotta, 261-283. 
SASSEN, Saskia

2001 "Spatialities and Tempralities of the Global: Elements for Theorization" en Arjun Appadurai (Ed.) Globalization Londres: Duke University Press, 260-277.

SCHUMPETER, Joseph A.

1957 Teoría del desenvolvimiento económico. México: Fondo de Cultura Económica, tercera edición.

SOLLEIRO, José Luis; LUNA, Katya; CASTAÑÓN, Rosario

2009 "Políticas públicas que sustentan los sistemas Regionales de innovación: aportes para la discusión” en, Daniel Villavicencio y Pedro Luis López de Alba (Coords.) México: Concyteg, Red Complejidad Ciencia y Sociedad, Conacyt, Editorial Plaza y Valdés.

TEECE, David; PISANO, Gary

1994 Dynamic capabilities of firms: an introduction". Industrial and corporate change, Oxford, vol 3, issue 3: 537-556.

TSING, Anna.

2005 “The Global Situation”. Jonathan Xavier y Renato Rosaldo (Eds.) The anthropology of globalization Blackwell, London.

VILLAVICENCIO, Daniel. y LÓPEZ DE ALBA, Pedro. coords.

2009 Sistemas de Innovación en México: regiones, redes y sectores. México: CONACyT / Plaza y Valdés editores 